\title{
On the Phase Noise Performance of Nonlinear Double-Loop Optoelectronic Microwave Oscillators
}

\author{
Romain Modeste Nguimdo, Yanne K. Chembo, Pere Colet and Laurent Larger
}

\begin{abstract}
We consider an optoelectronic oscillator for ultrapure microwave generation with two nonlinearly coupled delay loops in which the output of one of the loops modulates the other. We introduce a suitable dynamical model and study the phase noise performance. Tuning the parameters of the second loop allows to reduce the detrimental effect of the multiplicative phase noise and damp delay-induced spurious peaks. Besides, the nonlinear dynamics allows for stable microwave emission with larger amplitude than a single loop system.
\end{abstract}

Index Terms-Optoelectronic devices, microwave generation, phase noise reduction.

\section{INTRODUCTION}

$\mathbf{I}$ $\mathrm{N}$ some applications such as in radar, time-frequency metrology and lightwave technology, microwaves with exceptional purity are needed. Optoelectronic oscillators (OEOs) are useful for these applications because they can convert continuous light energy into stable and spectrally pure microwave signals [1], [2], [3]. In such systems, the purity of microwave signal is achieved thanks to an optical fiber delay-line inserted into the feedback loop. The role of the delay is to store the energy providing a quality factor equal to $Q=2 \pi f_{m} T$, where $f_{m}$ is the microwave frequency and $T$ the delay induced by the optical fiber. A convenient way to evaluate the purity in systems is to measure its phase noise spectrum, which is directly connected to the oscillator performance. The main advantage of the OEO is its capability to generate ultrastable, spectrally pure microwaves with frequencies as large as $75 \mathrm{GHz}$, and with a phase noise lower than $-160 \mathrm{dBc} / \mathrm{Hz}$ at $10 \mathrm{kHz}$ [4]. Later, these studies were complemented with a nonlinear and stochastic dynamics approach which enabled to investigate theoretically the stability properties of OEOs, and also to predict phase noise performance [5], [6], [7].

To reduce the phase noise, the first requirement consists in optimizing the RF and optoelectronic devices. Techniques to further reduce the noise include noise filtering [8], noise-tocarrier ratio minimization [9], exploitation of time variance [10], use of coupled oscillators [11] and use of resonators having good quality factor [12]. In the optical case, microwave

R. M. Nguimdo (email: modeste@ifisc.uib-csic.es) and P. Colet are with the Instituto de Física Interdisciplinar y Sistemas Complejos IFISC (CSIC-UIB), E-07122 Palma de Mallorca, Spain.

Y. K. Chembo and L. Larger are with the Optics Department, FEMTO-ST Institute, 16 Route du Gray, 25030 Besançon cedex, France.

Financial support from MICINN, Spain, and Feder under Projects TEC2006-10009 (PhoDeCC), FIS2007-60327 (FISICOS) and TEC200914101 (DeCoDicA) and by EC Project PHOCUS (FP7-ICT-2009-C-240763) is acknowledged. R.M.N. also acknowledges the fellowship BES-2007-14627 under the FPI program of MICINN. generation based on external modulation [13], or using a dualwavelength laser [14] are other examples of devices generating low phase noise signals. Since the Q-factor increases with the delay one may expect that a long delay line improves the phase noise performance. However, for single loop configurations, the use of long delays is limited because the delay is also responsible for the appearance of very strong parasite ringcavity peaks at integer multiples of the round-trip frequency $\Omega_{T}=2 \pi / T$, which reduce the region of low phase noise.

Alternatives were proposed, consisting in adding the output of two loops with different time-delay in order to further lower the phase noise or to reduce the level of parasite ringcavity peaks [15], [16], [17]. For example, in ref. [17] the level of the spurious peaks was reduced by about $30 \mathrm{~dB}$ although they were not completely damped. Along the same line, Jiang et al. obtained $-130 \mathrm{dBc} / \mathrm{Hz}$ at $1 \mathrm{MHz}$, although the performance at $100 \mathrm{MHz}$ was only $-50 \mathrm{dBc} / \mathrm{Hz}$ [18]. Another interesting configuration consisting in two OEO loops with dual injection locking has been recently proposed in refs. [19], [20], achieving low phase noise and low spurs (up to $-130 \mathrm{dBc} / \mathrm{Hz}$ at $100 \mathrm{KHz}$ ). In all the above schemes the two loops are on equal foot and the coupling is linear.

Single-loop OEOs suffer from another severe limitation: increasing the gain the system becomes unstable leading to a modulation of the microwave amplitude and thus to a degradation of the spectral purity [5]. Here we introduce a novel double-loop configuration in which the output of one of the loops is used to modulate the other. This is a quite asymmetrical configuration in which the two loops play a different role and are coupled nonlinearly. We present a dynamical model and derive an amplitude equation that allows to determine the parameter region where pure microwaves are generated. By including suitable stochastic terms we determine the phase noise performance. We show that, besides reducing the phase noise spurious peaks as linearly coupled dual-loop OEOs, this system allows for stable microwave emission with larger amplitude than a single loop system. The outline is as follows: in Sec. II we describe the system. In Sec. III we derive the amplitude equations. In Sec. IV we determine the steady states associated to pure microwave emission while in Sec. V we study its stability. In Secs. VI and VII we introduce a stochastic amplitude equation and evaluate the phase noise spectra. Final remarks are given in Sec. VIII.

\section{SYSTEM DESCRIPTION}

We consider a dual feedback loop as shown in Fig. 1. A semiconductor laser (SL) injects light into a Mach-Zehnder 


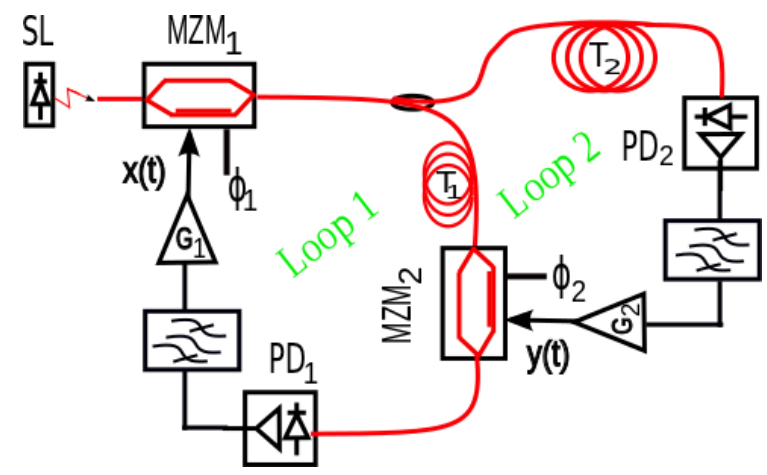

Fig. 1. Setup: SL: semiconductor laser, MZM: Mach-Zehnder modulator, PD: photodiode

modulator $\left(\mathrm{MZM}_{1}\right)$ which receives electrical voltages $V_{1}(t)$ and $V_{B, 1}$ in its radio-frequency (RF) and DC electrodes, respectively. Scaling those voltages respectively to the $\mathrm{RF}$ half-wave voltage $V_{\pi R F}$ and to the bias half-wave voltage $V_{\pi_{D C}}$ allows to define the dimensionless RF voltage $x(t)=$ $\pi V_{1}(t) /\left(2 V_{\pi R F}\right)$ and the offset phase $\phi_{1}=\pi V_{B, 1} /\left(2 V_{\pi_{D C}}\right)$. The complex envelope of the output electric field of $\mathrm{MZM}_{1}$ can be written as

$$
\mathcal{E}_{1}(t)=\frac{\mathcal{E}_{0}}{2}\left\{1+e^{2 i\left[x(t)+\phi_{1}\right]}\right\},
$$

where $\mathcal{E}_{0}$ is the SL output amplitude. Subsequently, $\mathcal{E}_{1}(t)$ is split into two parts. One part is delayed by a time $T_{2}$ and then converted into an electrical signal via the photodiode $\mathrm{PD}_{2}$. The electrical signal, proportional to $\left|\mathcal{E}_{1}\left(t-T_{2}\right)\right|^{2}$, is fed to a narrow-band filter with a central frequency $\Omega_{0}^{\prime}$ and $-3 \mathrm{~dB}$ bandwidth $\Delta \Omega_{2}$, amplified and applied as voltage $V_{2}(t)$ to the $\mathrm{MZM}_{2} \mathrm{RF}$ electrode. The narrow filter is expected to eliminate all the higher-order harmonics of the fundamental frequency $\Omega_{0}^{\prime}$. The second part of $\mathcal{E}_{1}(t)$ is delayed by a time $T_{2}$ and fed to $\mathrm{MZM}_{2}$ whose output field is

$$
\mathcal{E}_{2}(t)=\frac{\mathcal{E}_{1}\left(t-T_{1}\right)}{2}\left\{1+e^{2 i\left[y(t)+\phi_{2}\right]}\right\},
$$

with $y(t)=\pi V_{2}(t) /\left(2 V_{\pi R F}\right)$ and $\phi_{2}=\pi V_{B, 2} /\left(2 V_{\pi_{D C}}\right)$. The optical power $\left|\mathcal{E}_{2}(t)\right|^{2}$ is detected by $\mathrm{PD}_{1}$. The subsequent electrical signal is fed to another narrow band microwave $\mathrm{RF}$ filter of central frequency $\Omega_{0}$ with $-3 \mathrm{~dB}$ bandwidth $\Delta \Omega_{1}$ and then is connected to the $\mathrm{MZM}_{1} \mathrm{RF}$ electrode after amplification. In this scheme $\mathrm{MZM}_{1}$ optical output plays a double role: it is the optical input of $\mathrm{MZM}_{2}$ after a delay $T_{1}$, and it also used to drive $\mathrm{MZM}_{2}$ RF electrode after a delay $T_{2}$. Proceeding as in ref. [5], it can be shown that this system can be described by the dimensionless amplifier outputs $x(t)$ and $y(t)$ (see Fig. 1) as follows

$$
\begin{aligned}
& x+\frac{1}{\Delta \Omega_{1}} \frac{d x}{d t}+\frac{\Omega_{0}^{2}}{\Delta \Omega_{1}} u_{1}=\frac{\left|\mathcal{E}_{2}(t)\right|^{2}}{\left|\mathcal{E}_{0}\right|^{2}} G_{1} \\
& =\frac{G_{1}}{4}\left[F\left(x_{T_{1}}, \phi_{1}\right)+F\left(y, \phi_{2}\right)+F\left(x_{T_{1}}, \phi_{1}\right) F\left(y, \phi_{2}\right)+1\right], \\
& y+\frac{1}{\Delta \Omega_{2}} \frac{d y}{d t}+\frac{\Omega_{0}^{\prime 2}}{\Delta \Omega_{2}} u_{2}=\frac{\left|\mathcal{E}_{1}\left(t-T_{2}\right)\right|^{2}}{\left|\mathcal{E}_{0}\right|^{2}} G_{2} \\
& =\frac{G_{2}}{2}\left[F\left(x_{T_{2}}, \phi_{1}\right)+1\right],
\end{aligned}
$$

where $x_{t_{0}}=x\left(t-t_{0}\right), F(x, \phi)=\cos [2 x(t)+2 \phi], d u_{1} / d t=$ $x(t), d u_{2} / d t=y(t)$ and $G_{1}$ and $G_{2}$ are the overall loop gains (and do not only stand for the RF amplifier gains). The integral variables $u_{j}$ impose the mean value of $x(t)$ and $y(t)$ to be zero in the stationary regimes.

\section{Amplitude EQUATION}

We assume the filters to be narrowly resonant around the same central frequency $\Omega_{0}^{\prime}=\Omega_{0}$ so that all harmonics are filtered out. Considering

$$
u_{k}(t)=\frac{1}{2} \mathcal{U}_{k}(t) e^{i \Omega_{0} t}+c c,
$$

where $\mathcal{U}_{k}(t)$ is a slowly-varying amplitude and ' $c c$ ' stands for the complex conjugate of the preceding terms. Neglecting $\ddot{\mathcal{U}}_{k}$ as compared to $\Omega_{0} \dot{\mathcal{U}}_{k}$, where the dot stands for the derivative, the left-hand side (LHS) of Eqs. (3) and (4) can be written as

$$
\begin{aligned}
& \frac{d u_{k}}{d t}+\frac{1}{\Delta \Omega_{k}} \frac{d^{2} u_{k}}{d t^{2}}+\frac{\Omega_{0}^{2}}{\Delta \Omega_{k}} u_{k} \\
& \approx \frac{1}{2}\left[\left(1+2 i \frac{\Omega_{0}}{\Delta \Omega_{k}}\right) \dot{\mathcal{U}}_{k}(t)+i \Omega_{0} \mathcal{U}_{k}(t)\right] e^{i \Omega_{0} t}+c c .
\end{aligned}
$$

The variables $x(t)$ and $y(t)$ can also be written in terms of slowly-varying amplitudes $\mathcal{A}(t)=A(t) e^{i \psi(t)}$ and $\mathcal{B}(t)=$ $B(t) e^{i \varphi(t)}$ as

$$
\begin{aligned}
x(t) & =\frac{1}{2} \mathcal{A}(t) e^{i \Omega_{0} t}+c c \equiv A(t) \cos \left[\Omega_{0} t+\psi(t)\right], \\
y(t) & =\frac{1}{2} \mathcal{B}(t) e^{i \Omega_{0} t}+c c \equiv B(t) \cos \left[\Omega_{0} t+\varphi(t)\right] .
\end{aligned}
$$

By deriving Eq. (5), it turns out that $\mathcal{A}=\dot{\mathcal{U}}_{1}+i \Omega_{0} \mathcal{U}_{1} \simeq i \Omega_{0} \mathcal{U}_{1}$. Therefore the LHS of Eq. (3) can be written as

$$
\frac{1}{2}\left[\left(\frac{2}{\Delta \Omega_{1}}-\frac{i}{\Omega_{0}}\right) \dot{\mathcal{A}}(t)+\mathcal{A}(t)\right] e^{i \Omega_{0} t}+c c,
$$

and similarly for the LHS of Eq. (4) in terms of $\mathcal{B}$.

To derive the equations for the complex amplitudes $\mathcal{A}$ and $\mathcal{B}$ we have to consider the spectral components in $e^{i \Omega_{0} t}$ in the right-hand side (RHS) of Eqs. (3) and (4). For that purpose, as performed in ref. [6] for the single-loop case, we use the Jacobi-Anger expansion

$$
e^{i z \cos \alpha}=\sum_{q=-\infty}^{\infty} i^{q} \mathrm{~J}_{q}(z) e^{i q \alpha}
$$

where $\mathrm{J}_{q}$ are the $\mathrm{q}^{\text {th }}$-order Bessel functions of the first kind, with $q$ being an integer. As a consequence, we have

$$
F\left(x_{T}, \phi\right)=\frac{e^{2 i \phi}}{2} \sum_{q=-\infty}^{\infty} i^{q} \mathrm{~J}_{q}\left(2 A_{T}\right) e^{i q\left[\Omega_{0}(t-T)+\psi_{T}\right]}+c c
$$

and disregarding all the spectral components except the fundamental, we have

$$
F\left(x_{T}, \phi\right) \approx-\sin 2 \phi \mathrm{J}_{1}\left(2 A_{T}\right) e^{i\left(\Omega_{0} t-\Omega_{0} T+\psi_{T}\right)}+c c,
$$


and

$$
\begin{aligned}
& F\left(x_{T_{1}}, \phi_{1}\right) F\left(y, \phi_{2}\right) \approx-e^{i \Omega_{0} t}\left\{\cos 2 \phi_{1} \sin 2 \phi_{2} \sum_{p=-\infty}^{\infty} \mathcal{C}_{2 p}\right. \\
& \left.+\sin 2 \phi_{1} \cos 2 \phi_{2} \sum_{p=-\infty}^{\infty} \mathcal{C}_{1-2 p}\right\}+c c
\end{aligned}
$$

where

$$
\mathcal{C}_{q}=\mathrm{J}_{q}\left(2 A_{T_{1}}\right) \mathrm{J}_{1-q}(2 B) e^{i\left[-q \Omega_{0} T_{1}+q \psi_{T_{1}}+(1-q) \varphi\right]} .
$$

For the values of $A$ and $B$ considered here, Bessel functions of order 6 or larger can be neglected. Finally, collecting all fundamental terms, the equations for slowly-varying envelopes $\mathcal{A}(t)$ and $\mathcal{B}(t)$ can be written as

$$
\begin{aligned}
& \dot{\mathcal{A}}+\mu_{1} e^{i v_{1}} \mathcal{A}=-\frac{1}{2} \mu_{1} e^{i v_{1}} G_{1} \\
& \times\left\{\sin 2 \phi_{2} \mathrm{~J}_{1}(2 B) e^{i \varphi}+\sin 2 \phi_{1} \mathrm{~J}_{1}\left(2 A_{T_{1}}\right) e^{i \psi_{T_{1}}} e^{-i \Omega_{0} T_{1}}\right. \\
& \left.+\cos 2 \phi_{1} \sin 2 \phi_{2} \sum_{p=-2}^{2} \mathcal{C}_{2 p}+\sin 2 \phi_{1} \cos 2 \phi_{2} \sum_{p=-2}^{2} \mathcal{C}_{1-2 p}\right\}, \\
& \dot{\mathcal{B}}+\mu_{2} e^{i v_{2}} \mathcal{B}=-\mu_{2} e^{i v_{2}} G_{2} \sin 2 \phi_{1} \mathrm{~J}_{1}\left(2 A_{T_{2}}\right) e^{i\left(\psi_{T_{2}}-\Omega_{0} T_{2}\right)},
\end{aligned}
$$

where

$$
Q_{k}=\frac{\Omega_{0}}{\Delta \Omega_{k}} ; \mu_{k}=\frac{\Delta \Omega_{k}}{\sqrt{4+Q_{k}^{-2}}} ; v_{k}=\arctan \left[\frac{1}{2 Q_{k}}\right] \text {. }
$$

For oscillations to be sustained, phase matching conditions should be fulfilled, according to $e^{-i \Omega_{0} T_{k}}= \pm 1$. The matching condition $e^{-i \Omega_{0} T_{1}}=+1$ is equivalent to $e^{-i \Omega_{0} T_{1}}=-1$ by changing $\phi_{1}$ by $-\phi_{1}$ and $\varphi(t)$ by $\varphi(t)+\pi$. Similarly $e^{-i \Omega_{0} T_{2}}= \pm 1$ are equivalent changing $\phi_{2}$ by $-\phi_{2}$ and $\psi(t)$ by $\psi(t)+\pi$. Therefore without loss of generality we will consider $e^{-i \Omega_{0} T_{1}}=-1$ and $e^{-i \Omega_{0} T_{2}}=+1$.

In the particular case $G_{2}=0$, we straightforwardly have $\mathcal{B}=0$. Hence, since $\mathrm{J}_{q}(0)=0$ for $q \neq 0$, Eq. (15) is the same as for a single-loop OEO with gain $G_{1}\left(1+\cos 2 \phi_{2}\right) / 2$. In this case microwave emission takes place for $G_{1}>G_{1}^{t h}=$ $2 / \sin 2 \phi_{1}\left(1+\cos 2 \phi_{2}\right)$ [6].

It is interesting to note that for an offset phase $\phi= \pm \pi / 4$, we typically have an operation of the Mach-Zehnder modulator around the inflection point (thus leading to a linear modulation for small RF drive amplitude), or a cubic one when the latter is becoming closer or even greater than $V_{\pi R F}$, whereas for $\phi=0$ or $\pm \pi / 2$, a parabolic rest point is concerned. This is one of the physical justification of the nonlinear character of this double-loop OEO.

Since off-set phases appear in Eqs. (15) and (16) as $2 \phi_{1}$ and $2 \phi_{2}$ without loss of generality in the rest of the paper we will consider only the interval $[-\pi / 2, \pi / 2]$ for both phases.

\section{STEADY-STATE SOLUTIONS}

Our system is nonlinear, therefore for different parameter values it can have different number of stationary solutions. Pure microwave emission in both loops corresponds to a nonzero steady-state solution for $A$ and $B$. In this section we determine the parameter values for which this solution exist. Note that even if this solution exists, it may still be unstable, as we will discuss in the next section.

The steady state amplitudes $\left(A^{s t}\right.$ and $\left.B^{s t}\right)$ and phases $\left(\psi^{s t}\right.$ and $\varphi^{s t}$ ) of the microwaves are obtained setting $\dot{\mathcal{A}}=\dot{\mathcal{B}}=$ $\dot{\psi}=\dot{\varphi} \equiv 0$ in Eqs. (15) and (16). From Eq. (16), we obtain the following relationship

$$
B^{s t}=-G_{2} \sin 2 \phi_{1} \mathrm{~J}_{1}\left(2 A^{s t}\right) e^{i\left(\psi^{s t}-\varphi^{s t}\right)} .
$$

Since $B^{s t}$ is a real positive quantity, the imaginary exponent has to be either 0 or $\pi$. Hence there are two possibilities for the relative phase between the two microwaves: $\psi^{s t}=\varphi^{s t}$ (Case I) corresponding to the emission of two in-phase microwaves and $\psi^{s t}=\varphi^{s t}+\pi$ (Case II) corresponding to the emission of two out-of-phase microwaves. For typical values of $A^{s t}, \mathrm{~J}_{1}\left(2 A^{s t}\right)>0$, so $B^{s t}>0$ requires $\phi_{1} \in[-\pi / 2,0]$ for Case I and $\phi_{1} \in[0, \pi / 2]$ for Case II.

From Eq. (15) we have

$$
A^{s t}=\frac{G_{1}}{2} \mathrm{H}_{ \pm}\left(\phi_{1}, \phi_{2}\right)
$$

with

$$
\begin{aligned}
& \mathrm{H}_{ \pm}\left(\phi_{1}, \phi_{2}\right)=\sin 2 \phi_{1} \mathrm{~J}_{1}\left(2 A^{s t}\right) \mp \sin 2 \phi_{2} \mathrm{~J}_{1}\left(2 B^{s t}\right) \\
& \mp \cos 2 \phi_{1} \sin 2 \phi_{2} \sum_{p=-2}^{2} \mathrm{~J}_{2 p}\left(2 A^{s t}\right) \mathrm{J}_{1-2 p}\left(2 B^{s t}\right) \\
& +\sin 2 \phi_{1} \cos 2 \phi_{2} \sum_{p=-2}^{2} \mathrm{~J}_{1-2 p}\left(2 A^{s t}\right) \mathrm{J}_{2 p}\left(2 B^{s t}\right) .
\end{aligned}
$$

Here $\mathrm{H}_{+}$corresponds to Case $\mathrm{I}$ while $\mathrm{H}_{-}$corresponds to Case II. Since $\mathrm{H}_{-}\left(\phi_{1}, \phi_{2}\right)=\mathrm{H}_{+}\left(\phi_{1},-\phi_{2}\right)$, the steady state solutions satisfying $\psi^{\text {st }}=\varphi^{\text {st }}$ (Case I) are exactly the same as those obtained for Case II with the opposite $\phi_{2}$. However, for Case I, only the solutions with $\phi_{1} \in[-\pi / 2,0]$ are physical while for Case II, the physical solutions correspond to $\phi_{1} \in[0, \pi / 2]$.

The zero solution $A^{s t}=B^{s t}=0$ exists for all parameter values, although it may be unstable. This solution corresponds to zero RF voltage applied to $\mathrm{MZM}_{1}$ and $\mathrm{MZM}_{2}$. Thus, no microwave is generated, namely, the OEO does not oscillate. In a real OEO the emitted microwave will have a negligible amplitude (noise amplitude). Besides, a non-zero solution corresponding to pure microwave emission can exist depending on loop gains $G_{1}$ and $G_{2}$, and offset phases $\phi_{1}$ and $\phi_{2}$. Through an analytical approximation it is possible to estimate the contour of the parameter region where microwaves are emitted. Considering that $A^{s t}$ and $B^{s t}$ are small, Eqs. (18) and (19) can be expanded in series:

$$
A^{s t}=\frac{G_{1}}{2}\left[g_{1} A^{s t} \pm \frac{g_{2}}{2}\left(A^{s t}\right)^{3}\right],
$$

where + and - signs correspond to Case I and Case II respectively, and

$$
\begin{array}{r}
g_{1}=\sin 2 \phi_{1}\left[1+\cos 2 \phi_{2}+G_{2} \sin 2 \phi_{2}\left(1+\cos 2 \phi_{1}\right)\right], \\
g_{2}=\sin 2 \phi_{1}\left[1+\cos 2 \phi_{2}+G_{2} \sin 2 \phi_{2}\left(1+4 \cos 2 \phi_{1}\right)\right] \\
\sin ^{3} 2 \phi_{1}\left[3 G_{2}^{2} \cos 2 \phi_{2}+G_{2}^{3} \sin 2 \phi_{2}\left(1+\cos 2 \phi_{1}\right)\right] .
\end{array}
$$


(a)
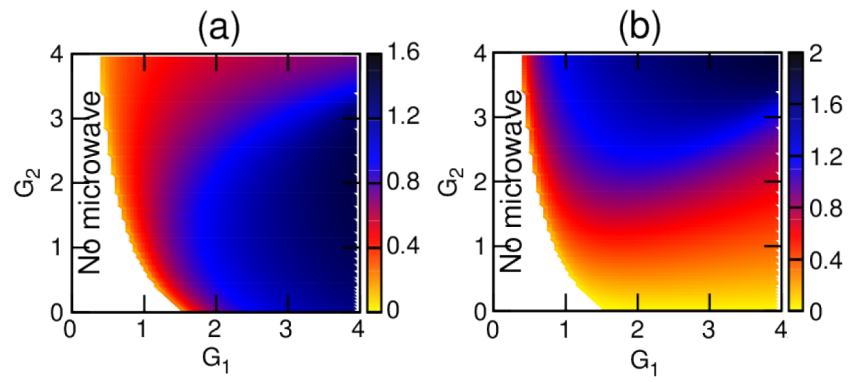

Fig. 2. Non-zero stationary solution given by Eqs. (18) and (19) with $\mathrm{H}_{-}$. (a) $A^{s t}$ and (b) $B^{s t}$ displayed in $\left(G_{1}, G_{2}\right)$-plane considering $\phi_{1}=0.5$ and $\phi_{2}=0.5$. (a)

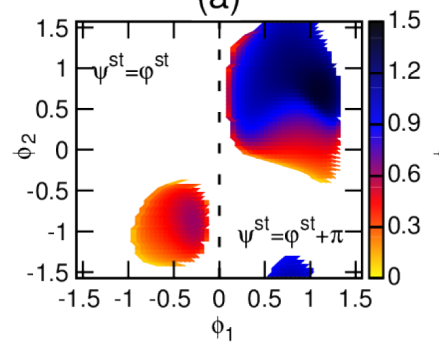

(b)

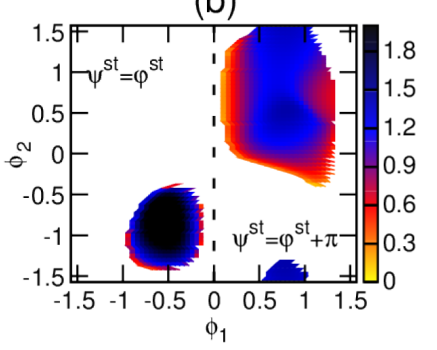

Fig. 3. Non-zero stationary solution given by Eqs. (18) and (19). (a) $A^{\text {st }}$ and (b) $B^{s t}$ displayed in $\left(\phi_{1}, \phi_{2}\right)$-plane considering $G_{1}=2.5$ and $G_{2}=2.5$.

From Eq. (21), one obtains the non-zero solution

$$
A^{s t}=\sqrt{\mp \frac{2}{g_{2}}\left(g_{1}-\frac{2}{G_{1}}\right)} .
$$

This solution exists only if the gain $G_{1}$ is larger than the threshold value $G_{1}^{t h}=2 / g_{1}$. The threshold for in- and out-ofphase microwave emission turns out to be the same. Note that for $\phi_{1} \in[-\pi / 2,0]$, the physical solution is in-phase while it is out-of-phase for $\phi_{1} \in[0,-\pi / 2]$.

For arbitrary values of the gains $G_{1}$ and $G_{2}$, Eqs. (18) and (19) are solved numerically to determine the stationary microwave amplitudes. Figure 2 displays in color scale the results for the out-of-phase microwave amplitude considering $\phi_{1}=0.5$ and $\phi_{2}=0.5$. For small values of $G_{1}$, there is no microwave emission. The range of values of $G_{1}$ for which the non-zero solution exists widens as $G_{2}$ increases. Besides the values of $A^{s t}$ and $B^{s t}$ can be as high as 1.2 and 2.0 [i.e, $\left|V_{1}(t)\right| \approx 0.76 V_{\pi R F}$ and $\left|V_{2}(t)\right| \approx 1.27 V_{\pi R F}$, respectively.

We now address the dependence of microwave emission as function of the off-set phases $\phi_{1}$ and $\phi_{2}$. Figure 3 displays in color scale the results for the in- and out-of-phase microwave amplitudes, considering $G_{1}=2.5$ and $G_{2}=2.5$. For $\phi_{1} \in[-\pi / 2,0]$, the physical solution is the in-phase one while it is out-of-phase for $\phi_{1} \in[0, \pi / 2]$. Depending on $\phi_{1}$ and $\phi_{2}$ there may be a strong microwave emission signaled by a large value for $A^{s t}$ and $B^{s t}$ or may not be emission at all. The large amplitudes are associated to an effective constructive interaction of the two delay loops while the lack of microwave emission is associated to destructive interaction. The contour of the region of microwave emission can be in principle determined by solving for $\phi_{1}$ and $\phi_{2}$ the threshold condition $g_{1} G_{1}=2$. However, we have found that for $\phi_{2}$ close to $\pi / 2$, the non-trivial solution appears sub-critically; therefore the region of existence of microwave emission shown in Fig. 3
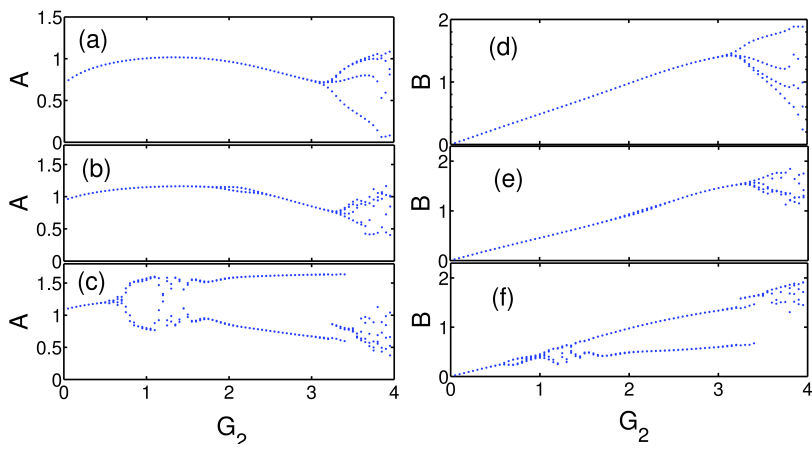

Fig. 4. Numerical bifurcation diagrams with respect to $G_{2}$ for $G_{1}=2$ (top row), $G_{1}=2.5$ (middle row) and $G_{1}=3.0$ (bottom row); considering $T_{1}=20 \mu$ s and $T_{2}=10 T_{1}$ and the initial conditions $A(0)=B(0)=0.2$ and $\psi(0)=\varphi(0)=0.5$. Other parameters are $\mu_{1}=\mu_{2}=50 \pi \mathrm{MHz}$, $Q_{1}=Q_{2}=200, \phi_{1}=0.5$ and $\phi_{2}=0.5$.

is slightly larger than the one obtained from the threshold condition. In the sub-critical region the non-zero steady state coexists with the zero solution.

\section{Numerical Simulations}

To check our analytical results and to investigate the stability of the steady state solutions shown in Figs. 2 and 3, Eqs. (15) and (16) are numerically integrated using a predictorcorrector method. For numerics, we consider $\mu_{1}=\mu_{2}=$ $50 \pi \mathrm{MHz}, Q_{1}=Q_{2}=200$ and $G_{1}=2.5$. The initial conditions are chosen so that, after the transient, the system converges to the physical solutions, i.e. $A \geq 0$ and $B \geq 0$.

Taking the loop gain $G_{2}$ as the bifurcation parameter and considering $\phi_{1}=\phi_{2}=0.5$, Fig. 4 displays bifurcation diagrams of the amplitude, obtained by extracting the maxima and the minima of the time series after the transient. For $G_{1}=$ 2 (upper row) the second-loop microwave amplitude grows practically linearly with $G_{2}$ while the first-loop one reaches a maxima at intermediate values of $G_{2}$. Therefore when $G_{2}$ is increased the second-loop emission becomes stronger than of the first loop. The system has stable microwave emission up to $G_{2} \approx 3$ as indicated by the fact that both $A$ and $B$ are singlevalued. Beyond this point the system undergoes an instability and $A$ and $B$ take several values for maxima and minima. Microwave emission at frequency $\Omega_{0}$ still takes place, but the amplitude is no longer constant, rather it is modulated. The modulation becomes more complex as $G_{2}$ is increased. For $G_{1}=2.5$ (middle row), the situation is similar, but there is an instability at $G_{2} \approx 1.8$ which leads to modulated amplitudes over a limited range of values for $G_{2}$. Then another stable region is found and finally modulated amplitudes develop for $G_{2}>3.2$. For $G_{1}=2.5$ (bottom row) the stable region is quite reduced since modulational instabilities appear already at $G_{2} \approx 0.6$.

Fig. 5 shows the numerical results obtained from Eqs. (15) and (16) for the stationary microwave amplitudes as function of $G_{1}$ and $G_{2}$ in the region where they are stable. Numerically we consider that the amplitude is stationary if after the transient time, it changes in less than $10^{-4}$ (allowing for numerical integration errors). The empty regions correspond to the values of $G_{1}$ and $G_{2}$ for which the microwave amplitude 
(a)
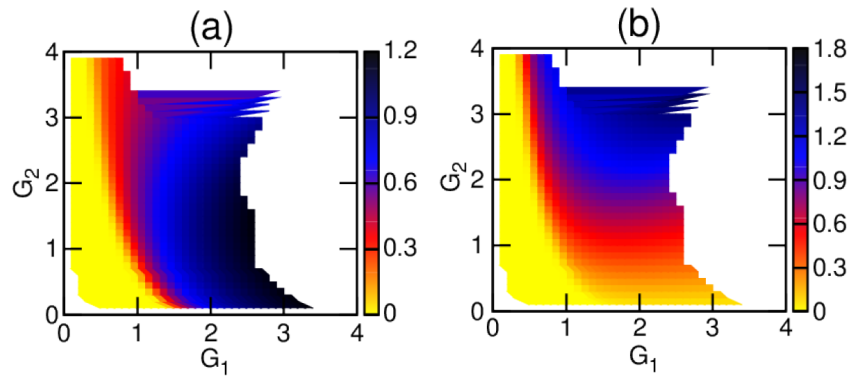

Fig. 5. Numerical results for the microwave amplitudes (a) $A^{\text {st }}$ and (b) $B^{\text {st }}$ considering $\phi_{1}=\phi_{2}=0.5$. The initial conditions and the parameters were set ac in Fir 4 The amnter recione sorrecenond to the godulated amnlitudes.
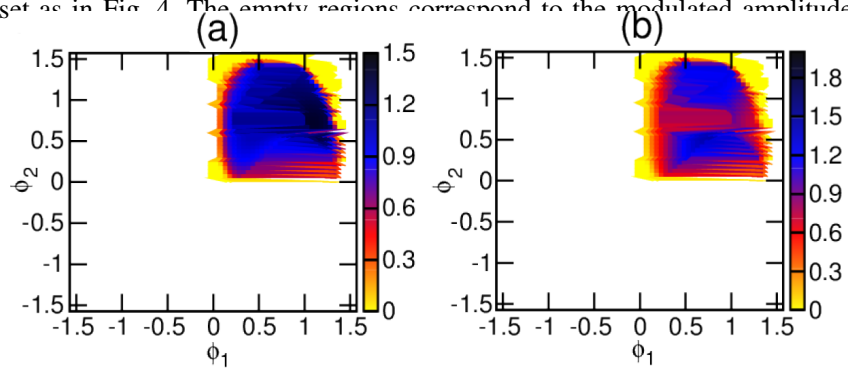

Fig. 6. Numerical results for the microwave amplitudes (a) $A^{s t}$ and (b) $B^{s t}$ as in Fig. 5 but considering $G_{1}=2.5$ and $G_{2}=2.5$

is modulated either periodically or in a more complex from. Remarkably enough, for the first-loop stable microwave can have an amplitude as high as 1.2 [i.e, $\left|V_{1}(t)\right| \approx 0.76 V_{\pi R F}$ ] while that of the second loop can be close to 1.8 [i.e, $\left.\left|V_{2}(t)\right| \approx 1.14 V_{\pi R F}\right]$. The double loop configuration therefore constitutes a potential way to generate microwaves with higher amplitudes than with single loop for which the maximum stable microwave amplitude is $\approx 0.76 V_{\pi R F}[6]$. These results are in excellent agreement with those predicted in Sec. IV (see Fig. 2). Note that the region where no microwaves are generated is represented in white in Fig. 2 and in bright gray (yellow in color) in Fig. 5. Finally, Fig. 6 displays the dependence of the microwave amplitude on the offset phases $\phi_{1}, \phi_{2}$ as obtained from the numerical integration of Eqs. (15) and (16).

In the rest of this article we will consider parameter values for which stable microwave emission takes place. Modulational instabilities of the microwave amplitude are associated to the on-set of side-band peaks in the spectra as for example those shown in Fig. 3 of Ref. [5] for a periodically modulated amplitude arising in a single-loop OEO. For an efficient operation one wants to avoid this regime since the spectral purity will be largely degraded.

\section{NOISE EFFECTS}

Fluctuations can be modeled by adding noise sources to the deterministic model for the microwave dynamics, as performed in [7]. Two main noise contributions are to be considered.

The first contribution is additive noise, which is the result of environmental fluctuations. For each loop, it is considered to be spectrally white, and around the central frequency $\Omega_{0}$ can be written as

$$
\tilde{\xi}(t)=\frac{1}{2} \xi(t) e^{i \Omega_{0} t}+\frac{1}{2} \xi^{*}(t) e^{-i \Omega_{0} t}
$$

where $\xi(t)$ is the complex Gaussian white noise with correlation $\left\langle\xi(t) \xi^{*}\left(t^{\prime}\right)\right\rangle=4 D_{A, B} \delta\left(t-t^{\prime}\right)$ corresponding to the density power spectrum $|\tilde{\xi}(\omega)|^{2}=2 D_{A, B}$, where subindex $A$ and $B$ stand for the first and second loop respectively.

The second contribution is multiplicative noise, resulting from overall gain fluctuations. It has, in general, a non trivial structure since it is composed from very different noise contributions. It can be assumed as flicker near the carrier and white above a certain knee-value as it has been observed in experiments [7]. It can be taken into account by adding a small perturbation term to the overall loop gain, e.g. $G+\delta G$. Thus considering a dimensionless multiplicative noise $\eta(t)=\delta G / G$, its empirical power density can be taken as

$$
|\eta(\omega)|^{2}=2 D_{m}\left[1+\frac{\Omega_{H}}{\omega+\Omega_{L}}\right]
$$

where $\Omega_{L}$ and $\Omega_{H}$ are the low and the high corner frequency, respectively.

Considering the phase matching $e^{-i \Omega_{0} T_{1}}=-1$ and $e^{-i \Omega_{0} T_{2}}=1$ the stochastic equations for slowly varying envelope $\mathcal{A}(t)$ and $\mathcal{B}(t)$ can be written as

$$
\begin{aligned}
& \dot{\mathcal{A}}+\mu_{1} e^{i v_{1}} \mathcal{A}=\mu_{1} e^{i v_{1}} \xi_{1}-\frac{1}{2} \mu_{1} e^{i v_{1}} G_{1}\left(1+\eta_{1}\right) \\
& \times\left\{\sin 2 \phi_{2} \mathrm{~J}_{1}(2 B) e^{i \varphi}-\sin 2 \phi_{1} \mathrm{~J}_{1}\left(2 A_{T_{1}}\right) e^{i \psi_{T_{1}}}\right. \\
& \left.+\cos 2 \phi_{1} \sin 2 \phi_{2} \sum_{p=-2}^{2} \mathcal{K}_{2 p}-\sin 2 \phi_{1} \cos 2 \phi_{2} \sum_{p=-2}^{2} \mathcal{K}_{1-2 p}\right\} \\
& \dot{\mathcal{B}}+\mu_{2} e^{i v_{2}} \mathcal{B}=-\mu_{2} e^{i v_{2}} G_{2}\left(1+\eta_{2}\right) \sin 2 \phi_{1} \mathrm{~J}_{1}\left(2 A_{T_{2}}\right) e^{i \psi_{T_{2}}} \\
& +\mu_{2} e^{i v_{2}} \xi_{2}
\end{aligned}
$$

where

$$
\mathcal{K}_{q}=\mathrm{J}_{q}\left(2 A_{T_{1}}\right) \mathrm{J}_{1-q}(2 B) e^{i\left[q \psi_{T_{1}}+(1-q) \varphi\right]} \equiv \mathrm{R}_{q}+i \mathrm{I}_{q} .
$$

\section{A. Stochastic phase equation}

Since $\mathcal{A}=A e^{i \psi}$ and $\mathcal{B}=B e^{i \varphi}$, the stochastic phase noise equations from Eqs. (27) and (28) can be written as

$$
\begin{aligned}
& \dot{\psi}+\mu_{1} \sin v_{1}=\frac{\zeta_{\psi}}{A}-\frac{\mu_{1} G_{1}}{2 A}\left[1+\eta_{1}(t)\right]\left\{\sin 2 \phi_{2} \mathrm{~J}_{1}(2 B)\right. \\
& \times \sin \left(v_{1}+\varphi-\psi\right)-\sin 2 \phi_{1} \mathrm{~J}_{1}\left(2 A_{T_{1}}\right) \sin \left(v_{1}+\psi_{T_{1}}-\psi\right) \\
& \left.+\sin 2 \phi_{1} \cos 2 \phi_{2} \sum_{p=-2}^{2} \mathrm{I}_{2 p}-\cos 2 \phi_{1} \sin 2 \phi_{2} \sum_{p=-2}^{2} \mathrm{I}_{1-2 p}\right\} \\
& \dot{\varphi}+\mu_{2} \sin v_{2}=\frac{\zeta_{\varphi}}{B}-\frac{\mu_{2} G_{2}}{B} \sin 2 \phi_{1}\left[1+\eta_{2}(t)\right] \\
& \times \mathrm{J}_{1}\left(2 A_{T_{2}}\right) \sin \left(v_{2}+\psi_{T_{2}}-\varphi\right),
\end{aligned}
$$

where $\zeta_{\psi}=\xi_{1, R e}(t) \sin \left(v_{1}-\psi\right)+\xi_{1, I m}(t) \cos \left(v_{1}-\psi\right)$ and $\zeta_{\varphi}=\xi_{2, R e}(t) \sin \left(v_{2}-\varphi\right)+\xi_{2, I m}(t) \cos \left(v_{2}-\varphi\right)$ are independent Gaussian white noises with variances $\left\langle\zeta_{\psi}(t) \zeta_{\psi}^{*}\left(t^{\prime}\right)\right\rangle=$ $2 D_{B} \delta\left(t-t^{\prime}\right)$ and $\left\langle\zeta_{\varphi}(t) \zeta_{\varphi}^{*}\left(t^{\prime}\right)\right\rangle=2 D_{B} \delta\left(t-t^{\prime}\right)$. The subindices $R e$ and $I m$ refer to the real and imaginary parts.

In the regime of stable microwave emission considered here the effects of the noise on the amplitude can be neglected and the amplitude can be taken as constant $A^{s t}$. Linearizing Eqs. (30) and (31) we get 


$$
\begin{aligned}
& \dot{\psi}=\mu_{1}\left[\alpha_{ \pm} \psi_{T_{1}}+\left(1-\alpha_{ \pm}\right) \varphi-\psi+\frac{1}{2 Q_{1}} \eta_{1}(t)+\frac{\zeta_{\psi}}{A^{s t}}\right] \\
& \dot{\varphi}=\mu_{2}\left[\left(\psi_{T_{2}}-\varphi\right)+\frac{1}{2 Q_{2}} \eta_{2}(t)+\frac{\zeta_{\varphi}}{B^{s t}}\right]
\end{aligned}
$$

with

$$
\begin{aligned}
& \alpha_{ \pm}=\frac{1}{\mathrm{H}_{ \pm}}\left\{-\sin 2 \phi_{1} \mathrm{~J}_{1}^{A}-5 \sin 2 \phi_{1} \cos 2 \phi_{2} \mathrm{~J}_{5}^{A} \mathrm{~J}_{4}^{B}\right. \\
& -\cos 2 \phi_{1} \sin 2 \phi_{2}\left(\mathrm{~J}_{1}^{A} \mathrm{~J}_{1}^{B}+9 \mathrm{~J}_{3}^{A} \mathrm{~J}_{3}^{B}\right) / B^{s t} \\
& \left.\mp 4 \sin 2 \phi_{1} \cos 2 \phi_{2}\left(\mathrm{~J}_{2}^{A} \mathrm{~J}_{2}^{B}+4 \mathrm{~J}_{4}^{A} \mathrm{~J}_{4}^{B}\right) / B^{s t}\right\},
\end{aligned}
$$

where $\mathrm{J}_{n}^{x}=\mathrm{J}_{n}\left[2 x^{s t}\right]$. Here again the signs $(+)$ and $(-)$ refer to the Case I and Case II, respectively. It is worth noting that for $B=0$ (single OEO loop), $\alpha_{ \pm}=1$ and Eq. (32) becomes the one obtained for single loop OEO.

\section{B. Phase noise spectrum}

Since Eqs. (32) and (33) are linear, the phase noise spectrum in the whole frequency range can be obtained from the squared modulus of its Fourier transform. Thus considering that all noise sources are uncorrelated, the Fourier transform $\Psi(\omega)$ of the phase $\psi(t)$ can be written as

$$
\begin{gathered}
|\Psi(\omega)|^{2}=\frac{\omega^{2}+\mu_{2}^{2}}{D}\left(\frac{\mu_{1}^{2}}{4 Q_{1}^{2}}\left|\eta_{1}(\omega)\right|^{2}+\frac{2 \mu_{1}^{2}}{A^{s t^{2}}} D_{A}\right) \\
+\frac{\mu_{1}^{2}\left(1-\alpha_{ \pm}\right)^{2}}{D}\left(\frac{\mu_{2}^{2}}{4 Q_{2}^{2}}\left|\eta_{2}(\omega)\right|^{2}+\frac{2 \mu_{2}^{2}}{B^{s t^{2}}} D_{B}\right),
\end{gathered}
$$

where $D=\mid\left[i \omega+\mu_{1}\left(1-\alpha_{ \pm} e^{-i \omega T_{1}}\right)\right]\left(i \omega+\mu_{2}\right)-\mu_{1} \mu_{2}(1-$ $\left.\alpha_{ \pm}\right)\left.e^{-i \omega\left(T_{1}+T_{2}\right)}\right|^{2}$. At this stage, we note that for $\alpha_{ \pm}=1$ (single loop OEO) one recovers the phase noise spectrum formula given by [7]:

$$
|\Psi(\omega)|^{2}=\frac{\frac{\mu_{1}^{2}}{4 Q_{1}^{2}}\left|\eta_{1}(\omega)\right|^{2}+\frac{2 \mu_{1}^{2}}{A^{s t 2}} D_{A}}{\left|i \omega+\mu_{1}\left(1-e^{-i \omega T}\right)\right|^{2}} .
$$

Equation (35) shows that the phase noise spectrum depends on the delay time, on the quality factor and also on the bandwidth. In the following, we investigate the performance of the system to phase noise when comparing with a single loop optoelectronic microwave oscillator. A way to do that consists in comparing the phase noise spectrum of the two systems.

\section{COMPARISON PHASE NOISE SPECTRA BETWEEN OEOS WITH SINGLE AND DOUBLE LOOP}

In addition to the parameters used in the previous sections, we also consider the parameters related to noise as $D_{m A}=D_{m B}=5.0 \times 10^{-11} \mathrm{rad}^{2} / \mathrm{Hz}, D_{A}=D_{B}=$ $9.0 \times 10^{-16} \mathrm{rad}^{2} / \mathrm{Hz}$. For comparison, Fig. 7 (a) displays the phase noise spectrum for a single loop OEO, as given by Eq. (36) and for a double loop [Eq. (35)]. For the single loop we take $G=2$ so that $A^{s t}=0.41\left[|V|=0.26 V_{\pi R F}\right]$ and consider two delays $T=20 \mu \mathrm{s}$ (4 km of fiber length) (dotted line) and $T=200 \mu \mathrm{s}$ (40 km of fiber length) (dashed line). For the double loop (solid line) we take $G_{1}=2.0$,
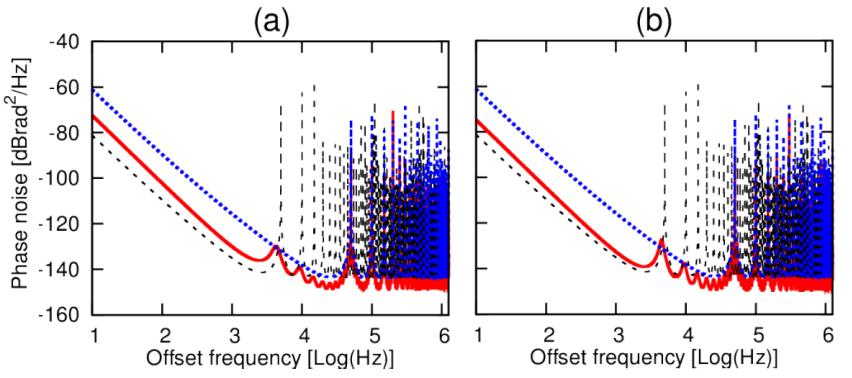

Fig. 7. Comparison of the theoretical phase noise spectrum of a singleloop OEO with $G=2$ and (dotted line) $T=20 \mu$ s and (dashed line) $T=200 \mu$ s with that of a double loop (solid line), both above threshold over a $500 \mathrm{MHz}$ spectral range analysis. The parameters for the double loop are $\phi_{1}=\phi_{2}=0.5, T_{1}=20 \mu \mathrm{s}, T_{2}=10 T_{1}, G_{1}=2.0$ and (a) $G_{2}=0.5$ and (b) $G_{\text {? }}=2.5$. (a)

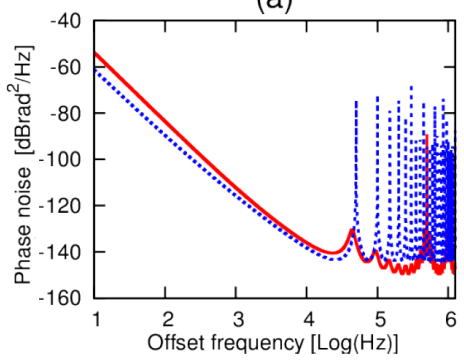

(b)

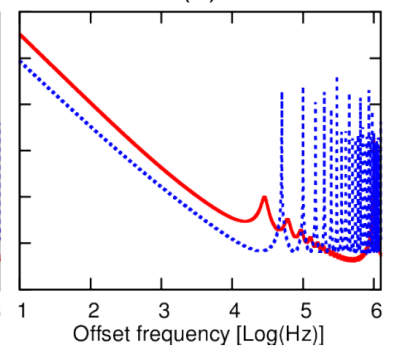

Fig. 8. Comparison of the theoretical phase noise spectrum of a single loop OEO with $G=2$ and $T=20 \mu$ s (dotted line) with that of a double loop (solid line), both above threshold in a $500 \mathrm{MHz}$. The parameters for the double loop are $\phi_{1}=\phi_{2}=0.5, G_{1}=2.0$ and (a) $T_{1}=2 \mu \mathrm{s}, T_{2}=10 T_{1}$ and $G_{2}=0.7$, (b) $T_{1}=1 \mu \mathrm{s}, T_{2}=30 T_{1}$ and $G_{2}=0.2$.

$G_{2}=0.5, T_{1}=20 \mu \mathrm{s}, T_{2}=10 T_{1}, \phi_{1}=\phi_{2}=0.5$ so that $A^{s t}=0.93$ and $B^{s t}=0.24\left[\left|V_{1}\right|=0.59 V_{\pi R F}\right.$ and $\left.\left|V_{2}\right|=0.15 V_{\pi R F}\right]$. It turns out that the phase noise is reduced about $14 \mathrm{~dB}$ for frequencies below $1 / T_{2}$. As the quality factor is proportional to the fiber length, the effect of the multiplicative noise is reduced [see Eq. (35)]. This leads to the phase noise performance observed in the region below $1 / T_{2}$ where the phase noise is dominated by multiplicative noise.

Interestingly enough the spurious peaks which would have appeared between $1 / T_{2}$ and $1 / T_{1}$ are damped due to interferences between the two combined signals in $\mathrm{MZM}_{2}$. Note that a small spurious peak still appears at $1 / T_{1}$ meaning that a residual constructive interference still exists at this frequency. Besides, the effect of the additive noise, which depends on $1 / A^{s t^{2}}$, is reduced in the double loop for which $A^{s t}$ is larger. For frequencies above $1 / T_{1}$, strong spurious peaks appear as in the single loop case. If the same delay line $(T=200 \mu \mathrm{s})$ were used in single loop, the performance of the system would have been compromised due to appearance of the spurious peaks between $1 / T_{2}$ and $1 / T_{1}$ [Fig. 7 (dashed line)].

When increasing $G_{2}=2.5$, which leads to microwave amplitudes $A^{s t}=0.86$ and $B^{s t}=1.22\left[\left|V_{1}\right|=0.55 V_{\pi R F}\right.$ and $\left.\left|V_{2}\right|=0.78 V_{\pi R F}\right]$, the phase noise is further reduced to about $18 \mathrm{~dB}$ below $1 / T_{2}$ [Fig. 7 (b)] getting closer to that obtained for a single loop OEO with $T=200 \mu$ s. We have found that as $G_{2}$ increases, the spurious peak amplitudes in the damping frequency region increase as well.

In second place, we reduce both delays in the double loop OEO by a factor 10 , so that $T_{1}=2 \mu$ s and $T_{2}=10 T_{1}$ keeping 


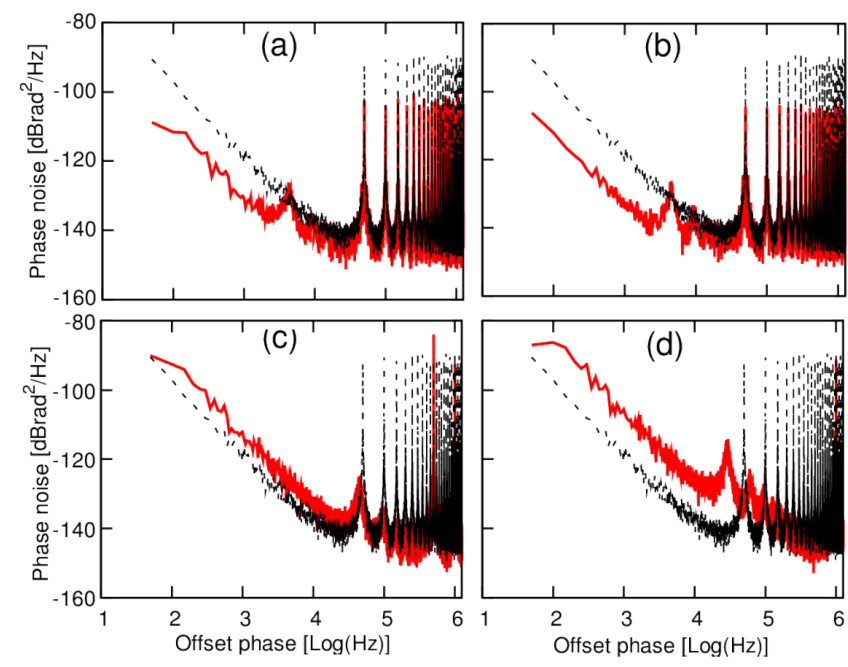

Fig. 9. Comparison of phase noise spectrum obtained from numerical simulation of stochastic amplitude equations for a single-loop OEO (dashed line) with $G=2$ and $T=20 \mu$ s with that of a double loop (solid line), both above threshold in a $500 \mathrm{MHz}$. The parameters for the double loop are $\phi_{1}=\phi_{2}=0.5, G_{1}=2$ and (a) $T_{1}=20 \mu \mathrm{s}, T_{2}=10 T_{1}$ and $G_{2}=0.5$ (b) $T_{1}=20 \mu \mathrm{s}, T_{2}=10 T_{1}$ and $G_{2}=2.5$, (c) $T_{1}=2 \mu \mathrm{s}, T_{2}=10 T_{1}$ and $G_{2}=0.7$, (d) $T_{1}=1 \mu \mathrm{s}, T_{2}=30 T_{1}$ and $G_{2}=0.2$. The spectrum is obtained by computing a time series of $0.08 \mathrm{~s}$ sampled into 10 equal parts.

$G_{1}=2.0$ and $\phi_{1}=\phi_{2}=0.5$ and considering $G_{2}=0.7$ (which leads to $A^{s t}=0.97,\left|V_{1}\right|=0.62 V_{\pi R F}$ and $B^{s t}=0.34$, $\left|V_{2}\right|=0.22 V_{\pi R F}$ ), and we compare the results with those obtained for the single loop OEO with delay $T_{1}=20 \mu \mathrm{s}$ and gain $G=2$ [Fig. 8(a)]. As in the previous case, the spurious peaks between $1 / T_{2}$ and $1 / T_{1}$ are suppressed. The first strong spurious peak appears around $1 / T_{2}=500 \mathrm{kHz}$. Therefore, the phase noise performance of the system is significantly improved since the same phase noise performance is obtained for single OEO loop but at $50 \mathrm{kHz}$. Better results can be achieved tuning the values of $T_{1}, T_{2}$ and $G_{2}$. For example Fig. 8(b) shows the results for $T_{1}=1 \mu \mathrm{s}, T_{2}=30 T_{1}$ and $G_{2}=0.2$ corresponding to $A^{s t}=0.82$ and $B^{s t}=0.1$ $\left[\left|V_{1}\right|=0.52 V_{\pi R F}\right.$ and $\left.\left|V_{2}\right|=0.06 V_{\pi R F}\right]$, again compared with the same single loop OEO. In that case, the phase noise floor is around $-150 \mathrm{dBrad}^{2} / \mathrm{Hz}$ at $1 \mathrm{MHz}$ and around $-50 \mathrm{dBrad}^{2} / \mathrm{Hz}$ at $10 \mathrm{~Hz}$.

While Figs. 7 and 8 plot only the phase noise for the first loop, we have found that the results for the phase noise in the second loop are very similar. In the region dominated by the multiplicative noise (frequencies below $1 / T_{2}$ ) they coincide. In the region dominated by the additive noise (frequencies above $1 / T_{1}$ ) for parameters such that $A^{s t}>B^{s t}$ (as we have mainly considered) the phase noise for the second loop is slightly larger than $\Psi(\omega)$.

In order to confirm these results, Eqs. (15) and (16) are directly simulated using the second order Runge-Kutta method for stochastic equations. These equations include noise both in phase and amplitude. For the sake of simplicity, the multiplicative noises are considered in simulations as Gaussian white instead of flicker ones, e.g. with spectral density $\left|\eta_{1,2}(\omega)\right|^{2}=2 D_{m A, B}$. Figure 9 displays the numerical phase noise spectrum results for the same cases as in Figs. 7 and 8 in comparison to a single loop OEO with $T_{1}=20 \mu \mathrm{s}$ and $G=2$. In Fig. 9 (a) and (b), the phase noise is considerably

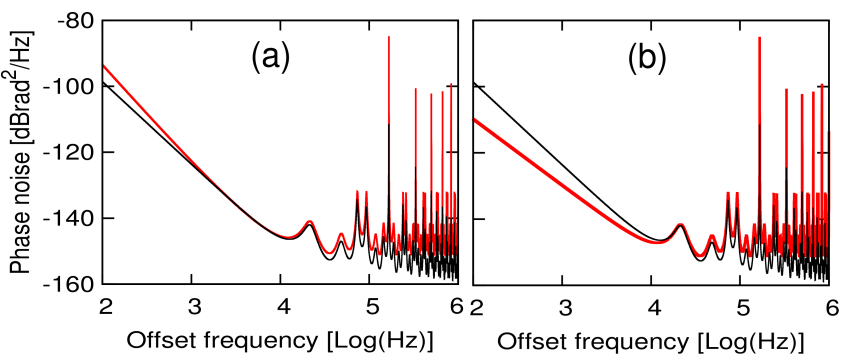

Fig. 10. Comparison of the phase noise spectrum obtained from Eq. (37) for $f_{0}=1.25 \mathrm{MHz}$ and after shifting of $-120 \mathrm{dBrad}^{2} / \mathrm{Hz}$ (black) with that obtained from Eq. (35) (gray, color) considering the multiplicative noise in Eq. (35) as (a) flicker, (b) white. The parameters are $T_{1}=12 \mu \mathrm{s}, T_{2}=42 \mu \mathrm{s}$, $G_{1}=G_{2}=2.2, \phi_{1}=\phi_{2}=0.5$.

reduced in the OEO system with double loop as already found analytically. For example one can see that the spurious peaks between $1 / T_{2}$ and $1 / T_{1}$ are damped while the strong spurious peaks above $1 / T_{1}$ emerge at the exact positions predicted from the analytical results [see Fig. 7 (a) and (b)]. Although the multiplicative noises considered in the simulations are white, it can be seen that, qualitatively speaking, the same conclusions as those predicted from analytics arise for all the cases. This therefore evidences that the improvements found in the double loop configuration do not depend on the nature of the noise.

On the other hand, the results in Fig. 9 (c) and (d) also evidence the possibility of extending the region of low phase noise up to $1 \mathrm{MHz}$ through the use of OEO system with double loop. As a final remark, note that the difference in the low frequency regime between the analytical and numerical results for the phase noise spectrum is due to the fact that we have considered a multiplicative noise with different spectra (flicker in the first case and white in the second).

We finally compare the phase noise performance of our nonlinear double loop OEO with that of the additive double loop considered by Eliyahu et al. [See Eq. (3) of [17]]:

$$
|\Psi(\omega)|^{2}=\omega^{-1 / 2}\left|1+\omega_{0}\left(\frac{e^{i \omega T_{1}}+e^{i \omega T_{2}}}{2\left(\omega_{0}-i \omega\right)}\right)\right|^{-2},
$$

where $\omega_{0}=2 \pi f_{0}, f_{0}$ being the filter's half width at halfmaximum transmission spectra. This is a phenomenological expression with no provision for different gain in both loops and which turns out to be independent of the microwave amplitude and offset phases. Figure 10 shows an example of phase noise spectra as reported in [17] (black) and that obtained from Eq. (35) (gray, color) with identical gain and offset phases for the two loops and considering the multiplicative noise as flicker (a) and white (b). As it can be seen, both spectra are similar when considering flicker noise spectrum, while for white multiplicative noise the results of the nonlinear system considered here are better.

\section{FINAL REMARKS}

We have introduced a double-loop configuration in which the output of one of the loops is used to modulate the other. While in previous double-loop OEOs both loops play a similar role and the coupling is linear, in our system the loops play a very different role and the coupling is nonlinear. We have derived an amplitude equation that allows to study the dynamics of the system and to determine the parameter regions where 
stable pure microwaves can be generated. Adding suitable noise sources to the amplitude equations allows to calculate the phase noise spectra. A similar treatment can be applied to other configurations for double-loop OEOs in order to better understand the dependence of the performance on the system parameters. As for the system considered here, besides reducing the phase noise spurious peaks as dual-loop OEOs considered before, it allows for stable microwave emission with larger amplitude than a single loop system.

\section{REFERENCES}

[1] X. S. Yao and L. Maleki, "High frequency optical subcarrier generator," Electronics Letters, 30 (18), 1525 (1994).

[2] X. S. Yao and L. Maleki, "Optoelectronic microwave oscillator," $J$. Opt.Soc. Amer. B 13, 1725, (1996).

[3] X. S. Yao and L. Maleki, "Optoelectronic oscillator for photonic systems," IEEE J. Quantum Electron. 32, 1141 (1996).

[4] See www.oewaves.com.

[5] Y. K. Chembo, L. Larger, H. Tavernier, R. Bendoula, E. Rubiola, and P. Colet, "Dynamic instabilities of microwaves generated with optoelectronic oscillators," Opt. Lett., 32, 2571 (2007).

[6] Y. K. Chembo, L. Larger, P. Colet, "Nonlinear dynamics and spectral stability of optoelectronic microwave oscillators," IEEE J. Quantum Electron. 44858 (2008).

[7] Y. K. Chembo, K. Volyanskiy, L. Larger, E. Rubiola, and P. Colet, "Determination of phase noise spectra in optoelectronic microwave oscillators: a Langevin approach," IEEE J. Quantum Electron., 45, 178 (2009).

[8] E. Hegazi, H. Sjoland, and A. A. Abidi, "A filtering technique to lower LC oscillator phase noise," IEEE Journal of Solid-State Circuits $\mathbf{3 6}$, 1921 (2001)

[9] D. Ham and A. Hajimiri, "Concepts and methods in optimization of integrated LC VCOs," IEEE Journal of Solid-State Circuits 36, 896 (2001).

[10] A. Hajimiri and T. H. Lee, "A general theory of phase noise in electrical oscillators," IEEE Solid-State Circuits 33, 179 (1998).

[11] J. Kim and B. Kim, "A low-phase-noise CMOSLC oscillator with a ring structure," ISSCC Digest of Technical Papers, 430 (2000).

[12] W. Andress and D. Ham, "A circular standing wave oscillator," ISSCC Digest of Technical Papers, 50 (2004).

[13] G. Qi, J. P. Yao, J. Seregelyi, S. Paquet, and C. Belisle, "Generation and distribution of a wide-band continuously tunable mm-wave signal with an optical external modulation technique," IEEE Trans. Microwave Theory Tech. 53, 3090 (2005).

[14] X. Chen, J. P. Yao, and Z. Deng, "Ultranarrow dual-transmission-band fiber Bragg grating filter and its application in a dual-wavelength singlelongitudinal-mode fiber ring laser," Opt. Lett. 30, 2068 (2005).

[15] X. S. Yao, L. Maleki, Y. Ji, G. Lutes, and M. Tu, "A dual-loop optoelectronic oscillator," Proc. IEEE Int. Freq. Contr. Symp., 545 (1998).

[16] L. Maleki, S. Yao, Y. Ji and V. Ilchenko, "New schemes for improved opto-electronic oscillator," In International Topical Meeting on Microwave Photonics, 1, 177, (1999).

[17] D. Eliyahu and L. Maleki, "Low Phase noise and spurious level in multi-loop opto-electronic oscillators," Proc. 2003 IEEE International Frequency Control Symposium and PDA Exhibition Jointly with the 17th European Frequency and Time Forum, 405 (2003).

[18] J. Yang, Y. Jin-Long, W. Yao-Tian, Z. Li-Tai, Y. En-Ze, "An optical domain combined dual-loop optoelectronic oscillator," IEEE Photon. Technol. Lett., 19, 807 (2007).

[19] E. C. Levy, O. Okusaga, M. Horowitz, C. R. Menyuk, W. Zhou, and G. M. Carter, "Comprehensive computational model of single- and dual-loop optoelectronic oscillators with experimental verification," Opt. Express 18, 21461 (2010).

[20] O. Okusaga, E. J. Adles, E. C. Levy, W. Zhou, G. M. Carter, C. R. Menyuk, and M. Horowitz, "Spurious mode reduction in dual injectionlocked optoelectronic oscillators," Opt. Express 19, 5839 (2011).

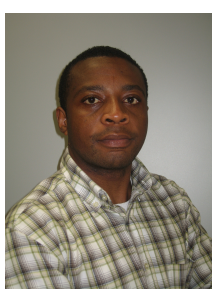

Romain Modeste Nguimdo was born in FongoTongo, Cameroon, on March 15, 1981. He received the M.Sc. degree in theoretical physics from the Universities of Dschang and Yaoundé I, Cameroon. He has just completed his Ph.D. degree at the Instituto de Física Interdisciplinar y Sistemas Complejos, IFISC (CSIC-UIB), Palma de Mallorca, Spain. His research interest involves nonlinear dynamics, optical chaos cryptography, ultra-pure microwaves.

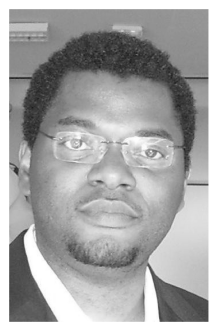

Yanne K. Chembo was born in 1976 at BlancMesnil, France. He received a Ph.D. degree in nonlinear dynamics in 2005 from the University of Yaoundé I, Cameroon, the Ph.D. degree in laser physics in 2006 from the Institute for CrossDisciplinary Physics and Complex Systems (formerly Mediterranean Institute for Advanced Studies), Palma de Mallorca, Spain, and the Habilitation degree in 2011 at the University of Franche-Comté, France. In 2007 and 2008, he was a postdoctoral fellow at the FEMTO-ST institute in Besanc on, France. In 2009, he has been awarded a NASA postdoctoral fellowship to undertake research on microwave photonics systems at the Jet Propulsion Laboratory (JPL/California Institute of Technology), Pasadena, USA. Since 2010, is a Senior Scientist at the Centre National de la Recherche Scientifique (CNRS), France. He has been a laureate of the ERC Starting Grant in 2011, and his research interests involve microwave photonics, optoelectronics, semiconductor laser physics, applied nonlinear and stochastic dynamics, and complex systems.

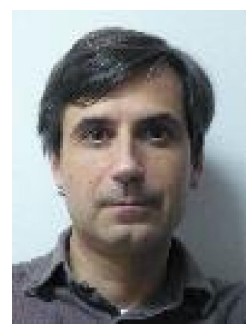

Pere Colet received the M.Sc. degree in physics in 1987 from the Universitat de Barcelona, and the Ph.D. degree in physics in 1991 from the Universitat de les Illes Balears, Palma de Mallorca, Spain. He was a Postdoctoral Fulbright Fellow at the School of Physics, Georgia Institute of Technology, Atlanta. In October 1994, he joined the Departament de Física, Universitat de les Illes Balears. Since May 1995, he has held a permanent research position at the Spanish Consejo Superior de Investigaciones Científicas. His present position is Research Professor at IFISC in Palma de Mallorca. He has co-authored over 110 publications in SCI journals. His research interests include fluctuations and nonlinear dynamics of semiconductor lasers, synchronization of chaotic lasers and encoded communications, synchronization of coupled nonlinear oscillators, pattern formation and quantum fluctuations in nonlinear optical cavities and dynamics of dissipative solitons.

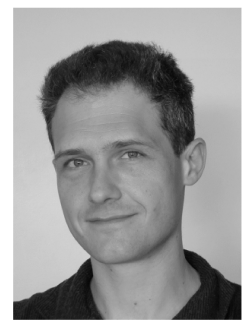

Laurent Larger received the degree in electronic engineering from the University of Paris XI, Orsay, France, in 1988, the Agrégation degree in applied physics in 1991, and the Ph.D. degree in optical engineering and the Habilitation degree from the University of Franche-Comté in 1997 and 2002. respectively. He was responsible from 2003 to 2006 of the international research center GTL-CNRS Telecom, a joint laboratory between the French CNRS Georgia Tech University of Atlanta, and the University of Franche-Comté. He got a full professor position at the University of Franche-Comté in 2005. His current research at the Franche Comté Electronique, Mécanique Thermique et Optique Sciences et Technologies (FEMTO-ST) Institute, Besançon, includes the study of chaos in optical and electronic systems for secure communications, delayed nonlinear dynamics, optical telecommunication systems, and high spectral purity optoelectronic oscillators. Since September 2007 he is a Junior member at the Institut Universitaire de France (IUF). 